\title{
Augmented Lagrangian Genetic Algorithm Based Decentralized Control Configuration Design for Fluid Catalytic Cracking Units
}

\author{
Dauda Olurotimi Araromi*, Kazzem Kolapo Salam, Aminah Abolore Sulayman \\ Department of Chemical Engineering, Ladoke Akintola University of Technology, Ogbomoso, Nigeria \\ Email: *doararomi@lautech.edu.ng
}

Received 18 September 2015; accepted 27 November 2015; published 30 November 2015

Copyright (C) 2016 by authors and Scientific Research Publishing Inc.

This work is licensed under the Creative Commons Attribution International License (CC BY). http://creativecommons.org/licenses/by/4.0/

c) (i) Open Access

\section{Abstract}

In this work, three decentralized control configuration designs-independent, sequential and simultaneous designs-were used in multivariable feedback configurations for PI control of the riser and regenerator temperatures of FCCU in order to compare their performances. Control design was formulated as optimization problem to minimize infinity norm of weighted sensitivity functions subject to $\mu$-interaction measure bound on diagonal complementary functions of the closed loop system. The optimization problem was solved using augmented Lagrangian genetic algorithm. Simulation results show that simultaneous and independent designs give good response with less overshoot and with no oscillation. Bound on $\mu$-interaction measure is satisfied for both designs meaning that their nominal stabilities are guaranteed; however, it is marginal for simultaneous design. Simultaneous design outperforms independent design in term of robust performance while independent design gives the best performance in terms of robust stability. Sequential design gives the worst performance out of the three designs.

\section{Keywords}

FCCU, PI Controller, Decentralized Control, Lagrangian, Genetic Algorithm, Robustness

\section{Introduction}

Fluid catalytic cracking (FCC) process is an added valued process that converts low-value crude heavy oil fractions into high-value light oil fractions of high quality fuels such as high-octane gasoline and olefins with the aid of a zeolite catalyst. Fluid catalytic cracking unit (FCCU) is the heart of the refinery because it is the primary

\footnotetext{
"Corresponding author.
} 
conversion process in the refinery. Between $40 \%$ and $50 \%$ of total gasoline produced from refinery is obtained through this process [1]-[3]. Its economic importance demands that its performance should always be improved upon in order to get the products at desired qualities and with minimal cost. FCCU is an integration of strongly coupled complex non linear processes such as riser reactor, regenerator and fractionators which are characterized with the complex chemical reactions mechanism, complex hydrodynamics and many operating constraints [3]-[5]. Great research efforts both from academic and industrial circles have been directed to development of FCCU focusing on improvement on catalysis of cracking process; modeling of complex dynamic behaviors of the unit with emphasis on bifurcation, steady state multiplicities and chaotic trend; both steady and dynamic optimization and advanced control.

Multi-facet nature of the process, strong interaction among its components, and complex dynamic behaviors of process result in difficulty in the design and implementation of its control structure. Several studies on the control of FCCU have been presented in open literature. Ansari \& Tade [6] developed a nonlinear constrained optimization strategy to design nonlinear multivariable control scheme in order to handle multiple constraints and interaction associated with FCC process. Jose, Jesus, \& Hector [1] designed a combined multivariable cascade control for composition regulation in FCCU. They used a pure integral control with composition measurements for primary or master control loop to provide servo responses to the secondary or slave control loops represented as a linear multivariable PI temperature regulator. Both riser exit and regenerator cyclone temperature were considered as measurable variables in the design of the secondary or slave control loops. Raluca et al. [7] used quasi-infinite-horizon NMPC based on multiple shooting optimization algorithms to design an inferential control scheme in order to regulate product distribution from the fractionator of FCCU. Ahmed [8] focused his work on decoupling control in order to account for the high interaction associated with FCCU by designing a multivariable temperature regulator loop on the basis of lump kinetic models to reject disturbance from variation in feedstock composition. Iancu \& Agachi [4] studied the effect of main disturbance on the complex dynamic behavior of heat integrated real FCC plant from a Romanian refinery with the purpose to develop an optimal advanced control scheme for the plant. MPC strategy was developed to achieve improvement in operation, product quality and cost reduction of the plant.

There are many advanced control techniques which are "full blown" methods to handle MIMO system. They, however, lead to higher order state space controller which may be difficult to implement and many of them may not be able to handle plant with delay [9]. Decentralized control systems have been widely used in industries to handle MIMO plants because many of existing SISO procedures can be used to tune the control parameters to obtain simple low-order control structure with inherent robustness quality and ease of implementation [10]. Decentralized control system (DCS) consists of individual feedback controllers that connect a subset of the output variables with a subset of the manipulated variables without the subset being used by any other controller. Among the reasons why decentralized control system is used in practice include: possibility of turning each controller one at a time with a less modelling effort; ease of understanding by the operators; reducing the requirement for control links thereby permitting decentralized implementation; the parameters turned have a direct and "localized" effect, and it is less liable to uncertainty [11] [12]. One major requirement in DCS is integrity property, that is, as subsystem controllers are brought in and out of service the closed loop system should remain stable. Integrity property is better accessed through decentralized integral controllability (DIC). DIC is concerned with the possibility of detuning a decentralized controller with integral action in each loop without introducing instability. Steady state RGA is a useful tool to test for DIC. DCS is DIC if and only if diagonal elements of steady state RGA of process model are all positive [11]. The three designs used in decentralized control system are independent design, simultaneous design, and sequential design.

In independent design, each diagonal element of the model is used to design diagonal controllers without taking into consideration the off-diagonal interactions with the requirement that individual loop is stable when turning the loop. Independent design is made use of when the system model is decoupled in space. Interactive plant will require some element of decoupling to have effective design. The main advantages of this method are: it is tolerant to failure, that is, failure of one loop will not cause instability to the remaining part of the system and its design is not by "trial-and-error". However, information about the controllers in the other loops is not made use of because of the assumption of independent design thus, making the derived bound to be only sufficient and not necessary for robust performance. This puts limitation to its performance [13]. Sequential design involves design of diagonal controllers one at a time in sequence with the existing designed controllers put into effect thereby reducing the design to a scalar (SISO) problem. The problems associated with sequential design 
include: 1) failure tolerance is not ensured when "lower-level” loops fail; 2) the method most often proceeds by "trial-and-error" and there is no specific guideline on the order to follow for controller design for each loop to ensure robust performance of the whole system and; 3) the method relies heavily on the orders the loops are designed and how controller for each loop is designed [13]. In simultaneous design, the theoretically optimal method for decentralized control, involves optimization of some cost function to have simultaneous design of all diagonal controllers using the complete model of the process. The cost function in the design is the infinity norm of mixed-sensitivity functions based on the notion of robust mixed-sensitivity control to adjust controller parameters.

In this work, the three designs were applied to the control of riser-regenerator sub process of FCCU by formulating the weighted sensitivity function as optimization problem with some bounds as constraints so as to minimize its infinity norm in order to guarantee robustness in term of performance and stability of the closed loop system. Augmented Lagrangian technique in the frame of genetic algorithm was used to solve the constrained problem. The control structure takes into consideration the economic performance of the FCC process which depends on the product distribution inside the riser and constraints characterizing the plant operation. The control designs rely on a simple dynamical model of the FCC unit.

\section{FCC Unit Description}

A typical modern FCC unit is made up of riser reactor, regenerator, main fractionator, disengager, two catalyst standpipes (one for transportation of spent catalyst to regenerator and the other one for transportation of regenerated catalyst to riser reactor). In addition, there are many ancillaries like feed pre-heater, CO boiler, cyclones, air heater, catalyst cooler, air blower, wet gas compressor, expander, etc. which provide support to the main equipment. A schematic diagram of FCC unit is given in Figure 1. Pre treated Feedstock from different sources are first pretreated to removes impurities such as sulpurand then pre-heated to raise it temperature to desired

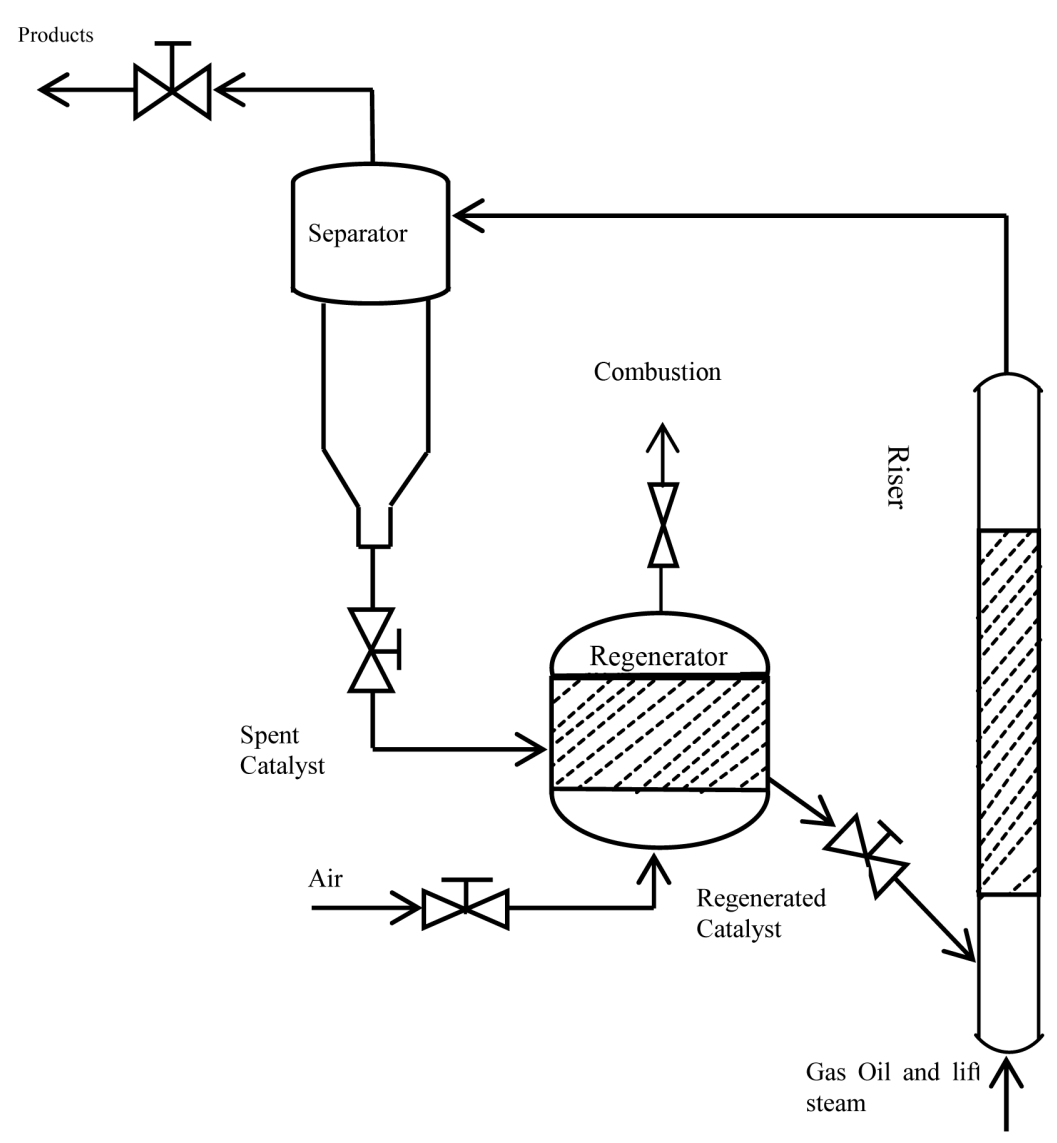

Figure 1. Diagrammatic sketch of a typical FCCU. 
value before it is passed to reactor. Riser reactor is made up of a vertical pipe whose diameter increases at certain height to accommodate increase in velocity of rising vapor. The velocity of vapor is around $6 \mathrm{~m} / \mathrm{s}$ at the base of the riser and increases to over $20 \mathrm{~m} / \mathrm{s}$ at the riser exit. Pre-heated feedstock and hot catalyst from regenerated catalyst standpipe meet at feed section of riser where gasoil is vaporized with the help of heat content of the hot catalyst coming from regenerator. The ratio of catalyst to oil in the riser feed section is in the range of 4:1 to $9: 1$ by weight. The cracking reactions take place as the vapor and the entrained catalyst flow up the riser. The reaction is endothermic and fast with a typical residence time in the range $5-8 \mathrm{~s}$ [1]. The temperature of the riser drops with height and riser top temperature is in the range 750 - $820 \mathrm{~K}$ depending on the circulation rate of the hot regenerated catalyst.

Coke formed during cracking reaction is deposited on the active sites of catalyst and subsequently reduces the activity of the catalyst. At the top end of the riser, vapour products and spent catalyst are separated at riser termination device (RTD). The spent catalyst is directed to catalyst stripper and hydrocarbon vapor products are contained in reactor vessel for some brief period to provide disengagement space between RTD and cyclones. Steam is passed to the stripper so as to remove volatile hydrocarbons from the catalyst. The stripped hydrocarbon vapor products from reactor vessel is first flown into cyclone reactor to remove spent catalyst which are still entrained in it before is flown finally to main fractionators for separation of hydrocarbons mixture into different products such as diesel, gasoline based on specific boiling ranges. Spent catalyst from stripper is passed to the regenerator through spent catalyst standpipe.

Regenerator is a fluidized reactor where combustion reaction takes place to burn off deposited coke on the spent catalyst in order to reactivate the catalyst activity. An air blower with the help of air distributor near the bottom of the regenerator brings in fresh air to burn off the coke. Combustion, being an exothermic reaction, the heat generated in coke burning is used to raise the catalyst temperature which is subsequently used to supply heat needed to maintain endothermic cracking reaction in riser reactor. The flue gas from regenerator is passed through regenerator cyclones to remove entrained catalyst and return it to regenerator. Hot regenerated catalyst is passed to riser reactor through standpipe which has slide valve to control recirculation rate.

\section{Control of FCCU}

The essence of controlling any process is to guarantee economic plant operation without sacrificing quality product, safety in operation and compliance with environmental regulation. All these objectives can be met if the operating variables of the system are well defined and categorized. In control of FCCU there exists a nonlinear, complex dynamic behavior, multi-variable, where the control loops often interact strongly in addition to the variable constraints which accompany its operation [6] [14] [15]. Therefore, control scheme must be structured to accommodate these complex input-output interactions as well as robustness in the presence of operating non-linearity, and errors in models.

Key operating variables in FCCU are divided into constrained controlled variables, constrained manipulated variables and disturbance variables. The constrained variables are either hard equipment constraints or operational constraints. Hard constraints are physical limitation on equipment while operational constraints arise from economic, safety and environmental reasons [16]. Violation of these constraints must be avoided for economic and safe operations of the system. Common constraints are maximum regenerator cyclone temperature, maximum wet gas compressor capacity, maximum air blower capacity, combustion air flow, feed flow rate, etc. For example, for economic reason, FCC unit should operate at maximum feed throughput with a constant riser temperature that will not violate its metallurgical limit and other operating constraints. Also for environmental reason, the oxygen in the flue gas must be maintained at environmentally friendly level subject to regenerator cyclones temperature at its metallurgical limit [6]. The optimal operating point for an FCC lies at one or several constraints and the control structure which allows operation closest to the constraints is preferable.

When constraints are satisfied, economic performance of the process must not be overlooked. The economic performance of the FCC process depends on the product distribution which is a function of the reaction conditions inside the riser reactor. Since the reaction taking place inside the riser is endothermic, the variable which has significant effect on reaction conditions is temperature. C4-yield and olefins, gasoline octane and bromine number, cycle oil aromaticity, coke yield and gasoline yield are all depend on reactor temperature [15]. Enthalpies of Feedstock and regenerated catalyst contribute greatly to the reactor temperature which means that flow rate of either of the two can serve as manipulated variable to control reactor temperature. Feed preheating tem- 
perature can be set to a minimum value required for gas oil to be completely vaporized. Regenerated catalyst rate which adjusts catalyst-to-oil ratios that favor high conversion can serve as manipulated variable for reactor temperature control. A slide valve in the regenerated catalyst standpipe line adjusts this flow rate.

Regenerator performance is affected by its temperature. Regenerator serves to burn off coke deposited on the catalyst. The coke deposited on the catalyst must be completely burnt otherwise there will be residual accumulation of coke which will lead to loss in catalyst activities. Coke which is mainly carbon is converted to directly to carbon dioxide or in step from carbon to carbon monoxide and then from carbon monoxide to carbon dioxide. Also, hydrogen in the coke gets converted to water. All these reactions are exothermic with maximum heat generated from the conversion of carbon monoxide to carbon dioxide. The total heat released accounts for the temperature of the regenerator. Most of the heat released is absorbed by the catalyst. The combustion rate is affected by combustion air flow rate from the blower. The oxygen in the air which burns the coke must be supplied in sufficient amount to have complete removal of the coke. Insufficient oxygen supply can affect the material balance of the units thereby lowering conversion level and leading to a phenomenon called behind in burning. This phenomenon is noticed when regenerator temperature starts coming down. Meanwhile, too much air flowrate to the regenerator can increase the velocity of catalyst leading to incomplete combustion of the $\mathrm{CO}$ to $\mathrm{CO}_{2}$ in the dense phase of the catalyst bed thereby transferring the burning location to the dilute phase where the combustion reaction which is highly exothermic proceeds further. Much of the heat released in this location cannot be fully absorbed due to low catalyst density around this location leading to increase in temperature and exposing regenerator cyclones and the flue gas lines to very high temperatures with possible thermal damage to the cyclones and other hardware. This phenomenon is called afterburning. Abnormal combustion and excessive temperatures can be prevented if the regenerator temperature is closely controlled and monitored which is possible with regulation of air flow rate to the regenerator. Thus, the temperatures in both reactors must be kept at values just below the metallurgical limits of the equipment materials. A FCCU model with potential to accommodate the main dynamical aspects of process is required for the design of control strategy to achieve this requirement.

\section{Model Used for the Controller Design}

The model adopted in this work is that of [14]. It was reported by [14] that the recipe to acceptable control of FCCU lies in selecting the proper controlled variables for the linear model needed for deign of control scheme. In this work, riser temperature $\left(\mathrm{T}_{\mathrm{s}}\right)$ is controlled with catalyst circulation rate (Fs) as manipulated variable and regenerator temperature $T_{r}$ is controlled with air flow rate $\left(\mathrm{F}_{\mathrm{z}}\right)$ as manipulated variable. The multivariable transfer functions for riser reactor-regenerator sub process obtained followed three steps. The first step involved extraction of an input and output information that characterizes the sub process (reactor and regenerator) from the FCCU model simulation. The second step involved determination of the transfer function type for each channel, where a first order transfer function was found to approximately represent each channel. The last step was to determine process gain and time constant that describe the transfer function. The graphical method described by [17] was used to determine the parameters.

\section{Decentralized Control Configuration Design System}

\subsection{Problem Definition}

For a given general square $n \times n$ multivariable system $\boldsymbol{G}(s)$ that represents nominal transfer function of a plant, the transfer function matrix can be split into its diagonal matrix $\boldsymbol{G}_{d}$ and its off diagonal matrix $\boldsymbol{G}_{m}$ in such a way that

$$
\boldsymbol{G}=\boldsymbol{G}_{d}+\boldsymbol{G}_{m} .
$$

The system is to be controlled by a diagonal controller $\boldsymbol{K}_{s}$. Let the diagonal matrices for the closed-loop system for the nominal plant be defined as follows:

For sensitivity function:

$$
\check{\boldsymbol{S}}(s)=\left[\boldsymbol{I}+\boldsymbol{G}_{d}(s) \boldsymbol{K}_{s}(s)\right]^{-1}=\operatorname{diag}\left\{\breve{s}_{l}(s)\right\}=\operatorname{diag}\left\{\left(1+g_{l l}(s) k_{l}(s)\right)^{-1}\right\} .
$$

For complementary sensitivity function: 


$$
\check{\boldsymbol{T}}(s)=\boldsymbol{G}_{d}(s) \boldsymbol{K}_{s}\left[\boldsymbol{I}+\boldsymbol{G}_{d}(s) \boldsymbol{K}_{s}\right]^{-1}=\operatorname{diag}\left\{\breve{t}_{l}(s)\right\}=\operatorname{diag}\left\{\frac{g_{l l}(s) k_{l}(s)}{1+g_{l l}(s) k_{l}(s)}\right\} .
$$

However, because of the process interactions, the stability of this diagonal subsystem may not necessarily guarantee the stability of the overall closed-loop system defined by:

For sensitivity function:

$$
\boldsymbol{S}(s)=\left[\boldsymbol{I}+\boldsymbol{G}(s) \boldsymbol{K}_{s}(s)\right]^{-1} .
$$

For complementary sensitivity function:

$$
\boldsymbol{T}(s)=\boldsymbol{G}(s) \boldsymbol{K}_{s}(s)\left[\boldsymbol{I}+\boldsymbol{G}(s) \boldsymbol{K}_{s}(s)\right]^{-1} .
$$

\subsection{Plant with Uncertainty}

We considered the set of transfer function matrices $\boldsymbol{G}_{p}(s) \in \mathrm{II}=\boldsymbol{G}^{i}(s), i=1,2, \cdots, N$ which represent controlled plant in different operating regions. To accommodate robustness in our design, we described the same controlled plant in different operating regions by assuming that the magnitudes and phases of transfer functions at a specific frequency lie in a disk region around the nominal transfer function matrix $\boldsymbol{G}(s)$. The disc-shaped regions can be generated by additive, multiplicative input and output uncertainties [12]. Plant Input uncertainty is defined according to Figure 2 as

$$
\boldsymbol{\Pi}: \boldsymbol{G}_{p}(s)=\boldsymbol{G}(s)\left(\boldsymbol{I}+\boldsymbol{W}_{I}(s) \Delta_{I}(s)\right), \bar{\sigma}\left(\Delta_{I}(s)\right) \leq 1, \forall \omega
$$

where $W_{I}$ is the magnitude of the relative (multiplicative) uncertainty at the plant inputs.

$$
l_{I}(s)=\max _{\boldsymbol{G}_{p}(s) \in \boldsymbol{\Pi}} \bar{\sigma}\left(\boldsymbol{G}(s)^{-1}\left(\boldsymbol{G}_{p}-\boldsymbol{G}(s)\right)\right) ;\left|\boldsymbol{W}_{I}(s)\right| \geq l_{I}(s) \forall \omega
$$

In Equation (7) $\bar{\sigma}($.$) define the maximum singular value of corresponding matrix and \Delta(s)$ is the perturbation which gives $\bar{\sigma}\left(\Delta_{I}(s)\right) \leq 1$. The nominal model was obtained as the mean value parameter model from models in different operating regions.

The closed-loop transfer from inputs $\left[\begin{array}{c}\mathbf{u}_{\Delta} \\ \boldsymbol{w}\end{array}\right]$ to outputs $\left[\begin{array}{c}\boldsymbol{y}_{\Delta} \\ \mathbf{z}\end{array}\right]$ according to Figure 3 was derived as follows

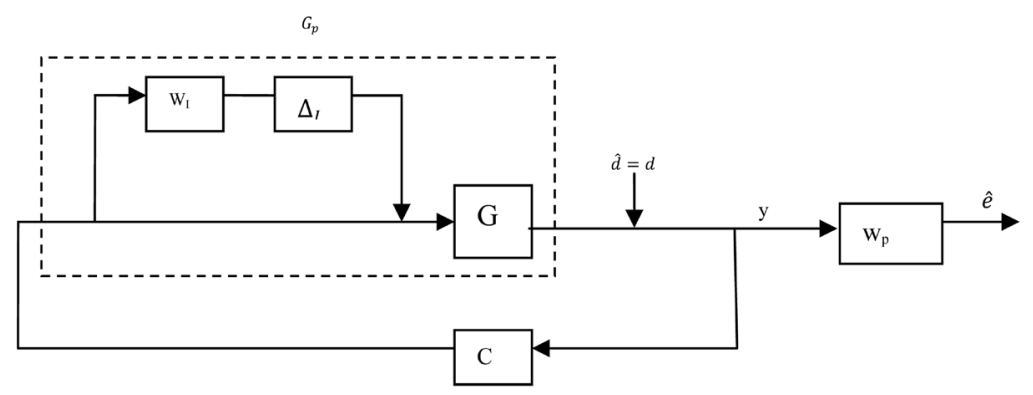

Figure 2. Plant with input uncertainty $\Delta_{I}(s)$ of magnitude $W_{I}(s)$.

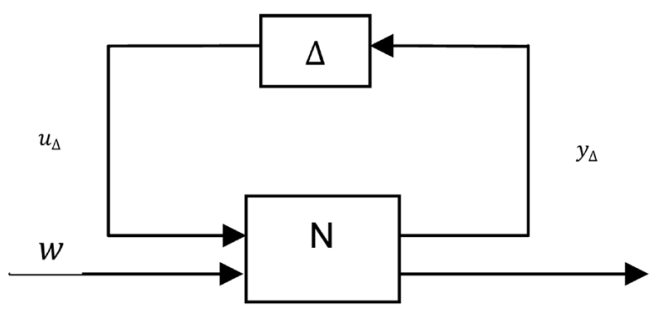

Figure 3. $N \Delta$-structure for robust performance analysis. 


$$
\left.\begin{array}{l}
\boldsymbol{y}_{\Delta}=\boldsymbol{W}_{I} \boldsymbol{u} \\
\mathbf{z}=\boldsymbol{W}_{p} \boldsymbol{w}+\boldsymbol{W}_{p} \boldsymbol{G} \boldsymbol{u}_{\Delta}+\boldsymbol{W}_{p} \boldsymbol{G} \mathbf{u}
\end{array}\right\} .
$$

But

$$
\boldsymbol{u}=\boldsymbol{K}_{\mathrm{s}} \boldsymbol{v}
$$

where $v$ is defined as

$$
\boldsymbol{v}=-\boldsymbol{w}-\boldsymbol{G} \boldsymbol{u}_{\Delta}-\boldsymbol{G} \boldsymbol{u} .
$$

Using (9) in (10) and with little algebraic manipulation we have

$$
\boldsymbol{u}=-\boldsymbol{K}_{\mathrm{s}}\left[\boldsymbol{I}+\boldsymbol{G} \boldsymbol{K}_{\mathrm{s}}\right]^{-1} \boldsymbol{w}-\boldsymbol{K}_{\mathrm{s}}\left[\boldsymbol{I}+\boldsymbol{G} \boldsymbol{K}_{\mathrm{s}}\right]^{-1} \boldsymbol{G} \boldsymbol{u}_{\Delta} .
$$

Using (11) in (8) and with little algebraic manipulation leads to

$$
N=\left[\begin{array}{cc}
-W_{I} K_{s} S G & -W_{I} K_{s} S \\
W_{P} S G & W_{P} S
\end{array}\right] .
$$

This is to imply that

$$
\left[\begin{array}{c}
\boldsymbol{y}_{\Delta} \\
\boldsymbol{z}
\end{array}\right]=\boldsymbol{N}\left[\begin{array}{c}
\boldsymbol{u}_{\Delta} \\
\boldsymbol{w}
\end{array}\right]=\left[\begin{array}{ll}
\boldsymbol{N}_{11} & \boldsymbol{N}_{12} \\
\boldsymbol{N}_{21} & \boldsymbol{N}_{22}
\end{array}\right]\left[\begin{array}{l}
\boldsymbol{u}_{\Delta} \\
\boldsymbol{w}
\end{array}\right]
$$

where $\boldsymbol{N}_{11}=-\boldsymbol{W}_{I} \boldsymbol{K}_{s}\left(\boldsymbol{I}-\boldsymbol{G} \boldsymbol{K}_{s}\right)^{-1} \boldsymbol{G}, \boldsymbol{N}_{12}=-\boldsymbol{W}_{I} \boldsymbol{K}_{s} \boldsymbol{S}, \boldsymbol{N}_{21}=\boldsymbol{W}_{P} \boldsymbol{S} \boldsymbol{G}$ and $\boldsymbol{N}_{22}=\boldsymbol{W}_{P} \boldsymbol{S}$.

In order to obtain the closed loop relationship between exogenous input vector $\boldsymbol{w}$ and exogenous output vector $\boldsymbol{z}$, we used the fact that $\boldsymbol{u}_{\Delta}=\Delta_{I} \boldsymbol{y}_{\Delta}$ from Figure 3 to eliminate $\boldsymbol{u}_{\Delta}$ and $\boldsymbol{y}_{\Delta}$ in (13) leading to

$$
F=F_{u}\left(N, \Delta_{I}\right) \triangleq N_{22}+N_{21} \Delta_{I}\left(I-N_{11} \Delta_{I}\right)^{-1} N_{12} .
$$

Requirement for stability and performance in term of $N \Delta$-structure shown in Figure 3 are summarized as follows [12]:

$$
\begin{aligned}
& N S \stackrel{\text { def }}{\Leftrightarrow} \boldsymbol{N} \text { is internally stable } \\
& N P \stackrel{\text { def }}{\Leftrightarrow}\left\|N_{22}\right\|_{\infty}<1 \text {; and } N S \\
& R S \stackrel{\text { def }}{\Leftrightarrow} F=F_{u}\left(N, \Delta_{I}\right) \text { is stable, } \forall \Delta_{I}\left\|\Delta_{I}(s)\right\|_{\infty} \leq 1 \text {; and } N S \\
& R P \stackrel{\text { def }}{\Leftrightarrow}\|F\|_{\infty}<1, \forall \Delta_{I}\left\|\Delta_{I}(s)\right\|_{\infty} \leq 1 \text {; and } N S
\end{aligned}
$$

A typical choice of $F$ is $\boldsymbol{W}_{P}\left(\boldsymbol{I}+\boldsymbol{G}_{p} \boldsymbol{K}\right)^{-1}$. It should be noted that Equation (15) indicates that all NP, RS and $\mathrm{RP}$ require NS to be satisfied.

\subsection{Nominal Stability}

Nominal stability is satisfied if $\boldsymbol{H}$ (and $\boldsymbol{S}$ ) is stable. Grosdidierand Morari (1986) introduced the $\mu$-interaction measure to give a sufficient condition for nominal stability (NS). In term of complementary sensitivity, assume $\check{\boldsymbol{T}}(s)$ is stable that is each loop is stable on its own, and that $\boldsymbol{G}$ and $\boldsymbol{G}_{m}$ have the same number of RHP (unstable) poles. Then $\boldsymbol{T}(s)$ is stable which means that the system is stable when all loops are closed according to

$$
N S \Leftarrow \max _{l}\left|\breve{t}_{l}\right| \leq \mu_{s}^{-1}\left(\boldsymbol{E}_{T}\right), \forall \omega, \boldsymbol{E}_{T}=\left(\boldsymbol{G}-\boldsymbol{G}_{d}\right) \boldsymbol{G}_{d}^{-1} .
$$

In order to permit integral action $(\check{\boldsymbol{T}}(0)=\boldsymbol{I})$, It is required that the system is diagonal dominant at low frequencies which implies that $\mu_{s}\left(\boldsymbol{E}_{T}\right)<1$ at $\omega=0$.

In term of sensitivity condition, for stable $\check{\boldsymbol{S}}(s)$ with $\boldsymbol{G}$ and $\boldsymbol{G}_{d}$ having the same number of RHP-zeros. Then $\boldsymbol{S}(s)$ is stable implying that the system is stable when all loops are closed according to [13]

$$
N S \Leftarrow \max _{l}\left|\breve{S}_{l}\right| \leq \mu_{s}^{-1}\left(\boldsymbol{E}_{S}\right), \forall \omega, \boldsymbol{E}_{S}=\left(\boldsymbol{G}-\boldsymbol{G}_{d}\right) \boldsymbol{G}^{-1} \text {. }
$$


For this condition to be satisfied and to ensure diagonal dominance at high frequencies, for any real system, it is required that $\check{\boldsymbol{S}}(s)=\boldsymbol{I}$ as $\omega \rightarrow 0$ and $\mu_{s}\left(\boldsymbol{E}_{S}\right)<1$ as $\omega \rightarrow 0$.

\subsection{Robust Stability (RS)}

Satisfying NS means that the stability of the system in Figure 3 is equivalent to the stability of the $M \Delta$-structure shown in Figure 4 where $M=N_{11}$ defines transfer function from the output to the input of the perturbation. From the definition of $\mathrm{N}_{11}$, sufficient and necessary condition RS is

$$
\mathrm{RS} \Leftrightarrow \mu(M(j \omega)) \bar{\sigma}(\Delta(j \omega))<\frac{1}{\bar{\sigma}\left(W_{I}(j \omega)\right)}, \forall \omega
$$

where

$$
\mu(M) \triangleq \frac{1}{\min \left\{k_{m} \mid \operatorname{det}\left(I-k_{m} M \Delta\right)=0 \text { for structured } \Delta, \bar{\sigma}(\Delta) \leq 1\right\}} .
$$

If no such structured $\Delta$ exists then $\mu(M)=0$.

A larger value of $\mu$ is bad as it implies that a smaller perturbation makes $\operatorname{det}(I-M \Delta)=0$, whereas a smaller value of $\mu$ is good.

\subsection{Performance Weight, $W_{P}$ Selection}

A given time domain performance specifications requirements can be roughly determined in frequency domain in terms of the bandwidth $\omega_{b}$ and the peak sensitivity $M_{s}$. In order to meet the closed-loop performance requirements for good control tracking of output variables, resulting sensitivity function $S$ should satisfy both the bandwidth $\omega_{b 1}$ and the peak sensitivity $M_{s}$ requirements. These requirements can be approximately represented in $W_{P}$ as

$$
W_{P}=\left[\begin{array}{cc}
\frac{s / M_{s 1}+\omega_{b 1}}{s+\omega_{b 1} A_{1}} & 0 \\
0 & \frac{s / M_{s 2}+\omega_{b 2}}{s+\omega_{b 2} A_{2}}
\end{array}\right]
$$

The parameters contained in $\boldsymbol{W}_{p}$ can be determined as follows:

In time domain control analysis, the speed of response is quantified by rise time $t_{r}$, settling time $t_{s}$ and quality of response is measured by overshoot $100 M_{p}$. These performance metrics can be approximately computed by using [18]:

$$
t_{r} \approx \frac{0.6+2.16 \xi}{\omega_{n}}, 0.3 \leq \xi \leq 0.8 ; t_{s} \approx \frac{4}{\xi \omega_{n}} ; M_{p}=\mathrm{e}^{-\frac{\pi \xi}{\sqrt{1-\xi^{2}}}}, 0<\xi<1 .
$$

With this, the speed of the response depends on $\omega_{n}$ while the overshoot depends on damping ratio $\xi$. The time domain specification $\omega_{n}$ and $\xi$ can be approximately related to frequency domain in term of bandwidth $\omega_{b}$ and the peak sensitivity function $M_{s}$ according to

$$
M_{i s}:=\left\|S_{i i}\right\|_{\infty}=\left|S\left(j \omega_{\max }\right)\right|=\frac{\alpha \sqrt{\alpha^{2}+4 \xi^{2}}}{\sqrt{\left(1-\alpha^{2}\right)^{2}+4 \xi^{2} \alpha^{2}}}
$$

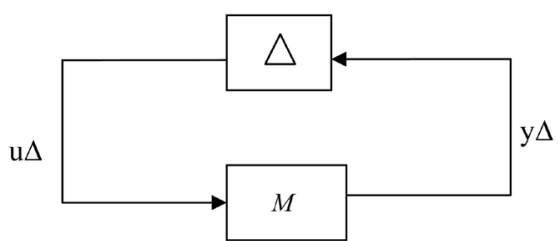

Figure 4. $M \Delta$-structure for robust stability analysis. 


$$
\alpha=\sqrt{0.5+0.5 \sqrt{1+8 \xi^{2}}}, \quad \omega_{\max }=\alpha \omega_{n} \text { and } \omega_{b} \approx \omega_{n} / \sqrt{2} .
$$

In Equation (20), $A_{i} \ll 1$ is selected to give approximate integral action in order to enforce zero steady state error in the closed loop response.

\subsection{Uncertainty Weight Selection}

Here, we want to hold stability and robust performance for the overall system in the presence in the presence of $20 \%$ uncertainty in both gains and time constants of the plant. We used multiplicative diagonal input uncertainty for the plant given by

$$
\boldsymbol{G}_{p}=\boldsymbol{G}\left[\boldsymbol{I}+\boldsymbol{W}_{I} \boldsymbol{\Delta}_{I}\right] ; \boldsymbol{\Delta}_{I}=\operatorname{diag}\left\{\delta_{i i}\right\}, \boldsymbol{W}_{I}=\operatorname{diag}\left\{w_{I_{i i}}\right\}
$$

where $W_{I}$ is the magnitude of the relative (multiplicative) uncertainty at the plant inputs bounded according to

$$
l_{I}=\max _{\boldsymbol{G}_{p} \in \Pi} \bar{\sigma}\left(\boldsymbol{G}^{-1}\left(\boldsymbol{G}_{p}-\boldsymbol{G}\right)\right) ;\left|\boldsymbol{W}_{I}\right| \geq l_{I i i} .
$$

\subsection{Nominal Plant and Issue of Input Constraints}

In this work, individual input and output variables were normalized with respect to their allowed range [19]. The normalized nominal transfer function matrix of the dynamic of the FCC unit was obtained as

$$
\boldsymbol{G}=\left[\begin{array}{cc}
\frac{2.700}{43.340 s+1} & \frac{0.105}{47.560 s+1} \\
\frac{-1.850}{23.760 s+1} & \frac{0.085}{26.115 s+1}
\end{array}\right]
$$

We employed $\underline{\sigma}(\boldsymbol{G})$ which is a measure of controllability of a system introduced by [19] to test for possibility of having input saturation when the system is under control. He established that for a properly scaled variables, a small value of $\underline{\sigma}(\boldsymbol{G})$ less than unity will require large input magnitude and such system is undesirable as this will lead to input saturation. The Thus to avoid input saturation, $\underline{\sigma}(\boldsymbol{G})$ must be larger than unity at all frequency. Examination of the nominal model indicates that $\underline{\sigma}(\boldsymbol{G})$ is less than unity at all frequencies implying that inputs constraints violation is possible with our plant when it is under control. We made effort to raise the value of $\underline{\sigma}(\boldsymbol{G})$ to be above unity by reshaping our plant. We selected the diagonal pre- and postcompensators $\boldsymbol{W}_{1}$ and $\boldsymbol{W}_{2}$ so that the singular values are desirable. $\boldsymbol{W}_{1}$ and $\boldsymbol{W}_{2}$ are of the form

$$
W_{1}=\left[\begin{array}{cc}
1 & 0 \\
0 & \frac{1}{1-\beta}
\end{array}\right], \quad W_{2}=\left[\begin{array}{cc}
1 & 0 \\
0 & \frac{1}{\beta}
\end{array}\right] \text { where } 0<\beta<1
$$

where $\beta$ is a design parameter which must be chosen to avoid input constraint violation and without inducing instability into the closed-loop system. Thus the shaped plant is now

$$
\boldsymbol{G}_{\mathrm{s}}=\boldsymbol{W}_{2} \boldsymbol{G} \boldsymbol{W}_{1} .
$$

The overall controller is given by

$$
\boldsymbol{K}=\boldsymbol{W}_{1} \boldsymbol{K}_{s} \boldsymbol{W}_{2} .
$$

\section{Augmented Lagrangian Optimization Technique}

\subsection{Optimization Technique}

Constrained optimization problems are often come across in engineering problems and the usual way of solving these kinds of problems is by using penalty function approaches. These approaches are relatively simple and easy to implement, however, they do not take into consideration the nature of mathematical formulation of the constraints at hand in term of linearity, nonlinearity, convexity, non-convexity, uni-modality and multi-modality. Also, penalty function approaches often lead to significant distortion of objective function thereby introducing non-linearity and multi-modalities which eventually create a bottleneck to optimization progress in arriving at 
true constrained optimization solution. In addition, they lack convergence proof but assume optimal solutions to the constrained optimization problem) [20].

In the recent past, augmented Lagrangian technique has received much attention as an efficient method to handle constrained optimization problems [20]-[22]. In augmented Lagrangian technique, a sequence of subproblems which are the outcome of combination of objective function and nonlinear constraint function are minimized while the linear constraints and bounds are not violated. The combination is achieved by using Lagrangian and the penalty parameters. The augmented Lagrangian technique is able to overcome problems associated with penalty function approaches and besides, it has the functionality to dynamically change the penalty parameters so as to move its optimum from unconstrained minimum point to the constrained minimum point with progress in iteration.

A general constrained optimization problem is usually expressed in the form

$$
\left.\begin{array}{r}
\underset{x \in \Omega}{\operatorname{Minimize}} f(\boldsymbol{x}) \\
\text { subject to } g_{i}(\boldsymbol{x}) \leq 0, \forall i=1,2, \cdots, l, \\
h_{j}(\boldsymbol{x})=0, \forall j=1,2, \cdots, m, \\
x_{k}^{\text {lower }} \leq x_{k} \leq x_{k}^{\text {upper }}, \forall k=1,2, \cdots, n .
\end{array}\right\}
$$

where $\boldsymbol{x}$ is an $n$ dimensional vector and $\Omega \subset \mathbb{R}^{n}\left(\Omega=\left\{\boldsymbol{x} \in \mathbb{R}^{n}: \boldsymbol{x}^{\text {lower }} \leq \boldsymbol{x}^{\text {upper }}\right\}\right), f(\boldsymbol{x})$ is the objective function, $h_{j}(\boldsymbol{x})=0, \forall j=1,2, \cdots, m$ are the equality constraints and $g_{i}(\boldsymbol{x}) \leq 0, \forall i=1,2, \cdots, l$ are inequality constraints.

\subsection{Augmented Lagrangian Method}

When augmented Lagrangian method is employed, the objective function is becomes an augmented Lagrangian that depends on a penalty parameter and on the multiplier vectors thus working like penalty functions. The augmented Lagrangian function takes the form [20].

$$
\mathcal{F}\left(\boldsymbol{x}, \lambda^{i t r}, \mu^{i t r}, \sigma^{i t r}\right)=f(\boldsymbol{x})+\sigma^{i t r} \sum_{i=1}^{l}\left[\max \left\{0, \lambda_{i}^{i t r}+\frac{g_{i}(\boldsymbol{x})}{\sigma^{i t r}}\right\}^{2}-\left(\lambda_{i}^{i t r}\right)^{2}\right]+\sigma^{i t r} \sum_{j=1}^{m}\left[\left(h_{j}(\boldsymbol{x})+\mu_{j}^{i t r}\right)^{2}-\left(\mu_{j}^{i t r}\right)^{2}\right]
$$

where $\sigma$ is a positive penalty parameter, $\lambda=\left(\lambda_{1}, \cdots, \lambda_{1}\right)^{\mathrm{T}}$ and $\mu=\left(\mu_{1}, \cdots, \mu_{m}\right)^{\mathrm{T}}$ are the Lagrangian multiplier vectors connected with the equality and inequality constraints respectively and with these $\mathcal{F}$ can penalize the solutions that want to violate equality and inequality constraints only. Here the index itr is the counter of the outer iterative process. Penalty parameter must tend to zero as the iteration is progressing in order to ensure global convergence. While iteration is in progress, Langrage multipliers $\lambda^{\text {itr }}$ and $\mu^{\text {itr }}$ are updated according to first order formulae

$$
\left.\begin{array}{l}
\lambda_{i}^{i t r+1}=\max \left\{0, \lambda_{i}^{i t r}+g_{i}\left(\boldsymbol{x}^{i t r}\right)\right\}, \forall i=1,2, \cdots, l \\
\mu_{j}^{i t r+1}=h_{j}\left(\boldsymbol{x}^{i t r}\right)+\mu_{j}^{i t r}, \forall j=1,2, \cdots, m
\end{array}\right\}
$$

Local convergence is enforced to a certain tolerance $\vartheta$ as defined in [22]

$$
\vartheta^{i t r}=\psi\left(1+\left\|\lambda^{i t r}\right\|+\left(\sigma^{i t r}\right)^{-1}\right)^{-1}, \psi>0 .
$$

The stopping criterion is based on an error function, $E\left(x, \lambda^{\text {itr }}\right)$ defined according to [22]

$$
E\left(x, \lambda^{i t r+1}\right)=\frac{\max \left\{\|g(x)\|_{\infty}, \max _{i} \lambda_{i}^{i t r+1}\left|g_{i}(x)\right|\right\}}{1+\left\|\lambda^{i t r+1}\right\|} .
$$

The algorithm will search for approximate solution to sub-problem (30) using natural selection optimization based technique called Genetic Algorithm (GA) and stop if the following criterion is met

$$
\vartheta^{i t r} \leq \vartheta^{*} \text { and } E\left(x, \lambda^{i t r+1}\right) \leq \varphi^{*}
$$

where $\vartheta^{*}$ and $\varphi^{*}$ are small positive constant values. Otherwise, the algorithm will stop after a maximum 
outer iteration count itr $^{*}$ is reached. In this works, $\vartheta^{*}$ and $\varphi^{*}$ were chosen as $10^{-12}$ and $10^{-6}$, respectively, while maximum iteration count $i^{*}{ }^{*}$ is $300, \psi=0.5$. In order to return a feasible approximation while using the GA, any computed approximation of $\boldsymbol{x}$ which fall outside the set $\Omega$ is adjusted component wise according to

$$
x_{i}= \begin{cases}x_{i}^{\text {lower }} & \text { if } x_{i}<x_{i}^{\text {lower }} \\ x_{i} & \text { if } x_{i}^{\text {lower }} \leq x_{i} \leq x_{i}^{\text {upper }} \\ x_{i}^{\text {upper }} & \text { if } x_{i}>x_{i}^{\text {upper }}\end{cases}
$$

\section{Genetic Algorithm}

Genetic Algorithm (GA) is a technique which provides solution to optimization problems by using survival of fitness paradigm from the theory of evolution in biology which is based on the mechanism of evolution, natural selection, and genetics. The operations such as selection, crossover, inheritance, mutation and elitism are used to operate on several values of decision variables in each iterating step to implement survival of the fittest strategy [23]. At each step, individuals are selected at random from the current population to become parents that are used to produce the children for the next generation. Over successive generations, the population "evolves" toward an optimal solution. Genetic algorithm can be applied to solve a variety of optimization problems that are not well suited for standard optimization algorithms, including problems in which the objective function is discontinuous, nondifferentiable, stochastic, or highly nonlinear [24].

In GA, the fitness function is the objective function to be optimized. The fitness function corresponds to the objective function of the sub problem. An individual is a vector of decision variables used to evaluate fitnessfunction. An individual is sometimes referred to as a genome and the vector entries (i.e. each decision variable) of an individual is called chromosome, and each of the bits needed to code that variable is a gene in the chromosome. A population is an array of individuals. The population is evolved over generations to produce better solutions to the problem. The evolution is performed using a set of stochastic genetic operators, which manipulate the genetic code used to represent the potential solutions. The value of the fitness function for an individual is the fitness value of that individual. For minimization problem, the best fitness value for a population is the smallest fitness value for any individual in the population. Some of the individuals in the current population that have lower fitness values are chosen as elite. These elite individuals are passed to the next population. Parents are a group of individuals in the current population who contribute their genes - the entries of their vectors - to their children. Individuals that have better fitness values become parents in the current generation. The next generation consists of Elite children, Crossover children and Mutationchildren.

The following summarizes how the genetic algorithm was employed for this work:

- GA starts with a random creation of initial population of specified size $\mathrm{N}_{\mathrm{p}}$. The $\mathrm{N}_{\mathrm{p}}$ specified for this work is 20 . The initial population was created to be well-dispersed and have individuals that are on the boundaries of the constraints and satisfy the bounds create populations. To create subsequent population, individuals in the current population were scored by computing theirs fitness values (raw scores) using sub problem in Equation (31). Rank fitness scaling was used to rank each individual on the basis of its raw scores. The rank of an individual is its position in the sorted scores. The rank of the best individual has the rank 1 , the next best is 2 , and so on.

- Selection of individuals on the basis of fitness as parents follows by using tournament method. In this method, each parent is selected by choosing individuals at random, the individuals with higher fitness values have higher chances to be selected. The number of individuals to be selected called tournament size $\mathrm{T}_{\mathrm{s}}$ was set to 4 throughout the simulation. Then the best individuals out of the set are chosen as parents. Parents in the current population with the best fitness values are considered as elite individuals and they are passed to the next population as elite children. Elite count specifies the number of elite individuals to be created. The other children are created by crossover (recombination) and mutation operations.

- Crossover involves exchange of information between two selected individuals so as to extract the best genes from different individuals thereby produces potentially superior child. Crossover fraction $\mathrm{n}_{\mathfrak{c}}$, which defines the fraction of the next generation that crossover will produce, was set to 0.8 . Single point method was used in crossover operation. In Single point method, a random integer $n$ is first chosen between 1 and number of variables, then vector entries numbered less than or equal to $n$ are selected from the first parent and genes numbered greater than $n$ from the second parent. These entries are concatenated to form the child. For example:

o $\quad$ p1 $=[$ a b c de f g h $]$ 
o $\quad \mathrm{p} 2=\left[\begin{array}{llllllll}1 & 2 & 3 & 4 & 5 & 6 & 7 & 8\end{array}\right]$

o random crossover point $=3$

o child = [a b c $\left.4 \begin{array}{llll}5 & 6 & 7 & 8\end{array}\right]$

- Mutation produces the remaining individuals in the next generation. Mutation involves random changes to the genetic coding of individual thereby adds to the diversity of a population with increasing likelihood that the algorithm will generate individuals with better fitness values. Gaussian distribution was used in mutation operation. In this method, a random number is taken from a Gaussian distribution centered on zero and added to each vector entry of an individual. The method is characterized by two parameters (shrink and scale). Scale parameter is used to control the fraction of gene's range to search and this determines the standard deviation at the first generation while the shrink parameter is used to control how standard deviation shrinks as generations advance. The value of 1 was used for both parameters and with this; the standard deviation can shrink to 0 linearly on getting to the last generation.

The augmented Lagrangian Genetic algorithm for solving sub problem (31) is presented as follows:

Algorithm: Augmented Lagrangian Genetic algorithm

Step 1: Define itr $=0, \lambda_{i}^{0}=0, \forall i=1,2, \cdots, l, \quad \tau=0.5, \varphi^{0}=10^{-2}, \varphi^{*}=10^{-6}, \quad \sigma_{\min }=10^{-10}, \vartheta^{*}=10^{-12}, \rho=0.5$, $k^{*}=50, N_{\text {iter }}=50$

Step 2: for certain tolerance $\vartheta^{i t r}$, find an approximate minimizer, $\mathbf{x}^{i t r+1}$

Step 2: for certain tolerance $\vartheta^{\text {itr }}$, fina an approximate minimize, $\boldsymbol{x}^{i t r+1}$ to subproblem (Equation (30)).

Initialize the GAs population points $z^{l} \in \mathrm{R}$, for $l=1,2, \cdots, N_{P}$ randomly, $k=0$;

Step2. 1: carry out the following opearations for genetic optimization;

Fitness value evalation operation; Selection operation; crossover operation;

mutation to improve diversity;

$\mathrm{x}^{i t r+1} \leftarrow \mathbf{z}_{\text {best }}^{k}$;

If $\left(\mathrm{x}^{\text {itr+1 }}<\mathrm{x}^{\text {lower }}\right)$ then

$\mathrm{x}^{\text {itr+1 }} \leftarrow \mathrm{x}^{\text {lower }}$

Endif

If $\left(\mathrm{x}^{\text {itr+1 }}>\mathrm{x}^{\text {upper }}\right)$ then

$\mathrm{x}^{i t r+1} \leftarrow \mathrm{x}^{\text {upper }}$

Endif

If $(k<2)$ then

$\{k \leftarrow k+1$

Go to Step 2.1

Elseif $\left(\left|\mathcal{F}\left(\mathbf{z}_{\text {best }}^{k}\right)-\mathcal{F}\left(\mathbf{z}_{\text {best }}^{k-1}\right)\right|>\vartheta^{i t r}\right)$ then

$k \leftarrow k+1$;

Go to step 2.1

Elseif $\left(k=k^{*}\right)$

Endif

step 3: Update penalty and Langrage constants

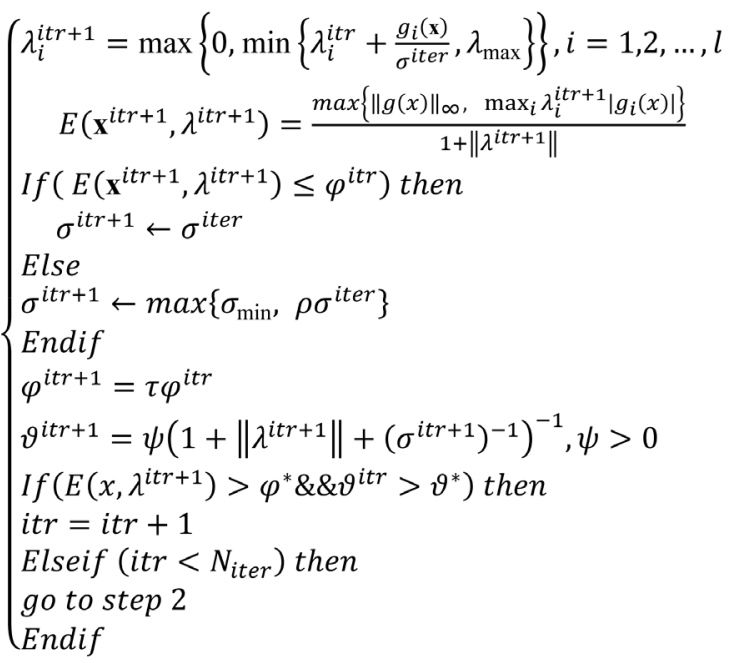




\section{Application of Augmented Lagrangian Genetic Algorithm to Control Design}

The algorithm was used in the design of decentralized controllers for the FCCU sub process. We considered the three different design techniques: independent design, sequential design and simultaneous design as follows:

\subsection{Independent Decentralized Control Design}

In independent design, we formulated an optimization problem such that

$$
\left\{\begin{array}{l}
\min _{k_{i}}\left|w_{P i}\left(I+g_{i i} k_{i}\right)^{-1}\right| \\
\text { subject to } \\
\left|\breve{t}_{i}\right| \leq\left|\left[\mu_{s}\left(E_{T}\right)\right]^{-1}\right|_{\infty}
\end{array}\right.
$$

where $w_{P i}$ is diagonal element of performance weight $\boldsymbol{W}_{P}, g_{i i}$ is diagonal elements of plant $\boldsymbol{G}$ and $\breve{t}_{i}$ is diagonal elements of complementary sensitivity function $\overrightarrow{\boldsymbol{T}}$. The controller $k_{i}$, whose parameters are to be determined by solving the optimization problem is used to close the loop $i$. The optimization problem was solved for each loop to obtain the overall controller $\boldsymbol{K}_{s}=\operatorname{diag}\left\{k_{i}\right\}$. Because of the assumption of independent designs, information about the other loops apart from diagonal loops of perturbed plant were not taken into consideration when designing each controller element. In order to have the overall control system behaves like independent SISO system, compensator is needed to decouple the loops. This was obtained by selecting pre-compensator $\boldsymbol{W}_{c}=[\boldsymbol{G}(0)]^{-1}$ in which $[\boldsymbol{G}(0)]$ is the gain matrix of the nominal plant [12].

\subsection{Sequential Decentralized Control Design}

Sequential design technique is suitable for interactive system loops with different response times. Sequential design of a decentralized controller $\left(\boldsymbol{K}_{s}\right)$ leads to a control system which is broken down both horizontally because $\boldsymbol{K}_{s}$ is diagonal and vertically because controllers for higher loops are tuned while lower loops are under control. In this method, multivariable nature of the process was ignored and a SISO controller was formulated for one pair of input and output variables. Once this design was successfully done the second SISO controller was designed for the second pair of variables while taking into consideration the effect of the controller around which feedback loop had already been closed. The loop with the fastest desired response times (“inner” loops) was first closed then followed by closing the "outer" loops that involved outputs with the slower desired response times.

We considered a square plant $\boldsymbol{G}$ with $\boldsymbol{y}=\boldsymbol{G} \boldsymbol{u}$, for outputs vector $\boldsymbol{y}$ which consist of $y_{1}$ and $y_{2}$ using input vector $\boldsymbol{u}$ which consist of $u_{1}$ and $u_{2}$. The control objective was achieved using a hierarchical control system. Controller $k_{2}$ was first designed to control the subset $y_{2}$ (fast control) followed by design of controller $k_{1}$ for output $y_{1}$ (slow control) while $k_{2}$ is in place (partially controlled system). Feedback control $u_{2}=K_{2}\left(r_{2}-y_{2}\right)$ was used to control subsystem involving $u_{2}$ and $y_{2}$. Elimination of $u_{2}$ and $y_{2}$ lead to the resulting partially controlled system:

$$
y_{1}=\left(g_{11}-g_{12} K_{2}\left(1-g_{22}\right)^{-1} g_{21}\right) u_{1} .
$$

We used the same optimization problem (37) presented under independent design to determine the parameters of $k_{i}$ for each loop.

\subsection{Simultaneous Decentralized Control Design}

The simultaneous design involves optimization of some cost function to have simultaneous design of all diagonal controller elements $k_{i}$ by using the complete model of the process. The design for controller $\boldsymbol{K}_{s}$ problem was expressed by the following optimization problem. Design a decentralized controller $\boldsymbol{K}_{s}$ such that

$$
\left\{\begin{array}{l}
\min _{K}\left\|\boldsymbol{W}_{P}\left(\boldsymbol{I}+\boldsymbol{G K}_{s}\right)^{-1}\right\|_{\infty} \\
\text { subject to } \\
\|\breve{\boldsymbol{T}}(s)\|_{\infty} \leq\left\|\left[\mu_{s}\left(\boldsymbol{E}_{T}\right)\right]^{-1}\right\|_{\infty}
\end{array}\right.
$$




\section{Simulation Result and Discussion}

Closed loop simulation was achieved with the following specification $\xi=0.4$ for each loop, $\omega_{n 1}=0.1448$ and $\omega_{n 2}=0.1281$. With these specifications we obtained $\omega_{b 1}=0.1024, \omega_{b 2}=0.0906, M_{1 s}=M_{2 s}=1.6242$ and we choose $A_{1}=A_{2}=10^{-5}$ and $\beta=0.1$ for all the designs and with the overall controller given by $\boldsymbol{K}=\boldsymbol{W}_{1} \boldsymbol{K}_{s} \boldsymbol{W}_{2}$.

Uncertainty bound weight $W_{I}$ was obtained as follows:

$$
\begin{gathered}
w_{I_{11}}=\frac{0.2523 s+0.003099}{s+0.02035} \\
w_{I_{22}}=\frac{0.9926 s+0.3301}{s+0.3321} .
\end{gathered}
$$

Table 1 shows PI controllers settings obtained for different designs using augmented Lagrange genetic algorithm optimization. Figure 2 shows the closed-loop performance of FCCU sub process for independent, simultaneous and sequential decentralized control designs. The set-point values used for riser temperature and regenerator temperature are respectively $[777.00 \mathrm{~K} 783.50 \mathrm{~K}]$ and $[988.00 \mathrm{~K} 993.00 \mathrm{~K}]$. Each set-point value was varied twice to observe persistence in performance. Total simulation time is 2500 mins with 500 mins for each set-point. To account for input constraints in the simulation we used ranges [0 kg/s $60 \mathrm{~kg} / \mathrm{s}]$ and [100 kg/s 400 $\mathrm{kg} / \mathrm{s}$ ] for air flow rate and catalyst flow rate, respectively. Table 2 presents the time domain performance metrics in terms of rise time, settling time and percentage overshoot. It was observed that simultaneous design gave the best performance in term of quality of response with the lowest overshoot for each loop. However, in term of speed of response, its response was a bit sluggish with large values both rise time and settling time when compared with the response observed from independent design. Both simultaneous and independent designs gave no oscillatory response. On the other hand, sequential design gave worst performance. Apart from the lowest rise time recorded for riser temperature response, the other performance metrics recorded indicated worst response when compared to other designs. As shown in Figure 2, he closed loop response with sequential controller was also characterized with oscillatory behavious and with the inverse response in regenerator temperature. Figure 2 also showed that as both outputs are step up simultaneously there are decrease in catalyst flow rate and increase in air flow rate and when they are step down the reverse is the case for catalyst and air flow rates. In all the three control schemes, only the sequential control violates air flow rate constraint.

The closed loop performances was examined further by using classical gain and phase margins analyses.

The bound $\left[\mu_{s}\left(\boldsymbol{E}_{T}\right)\right]^{-1}$ on $\bar{\sigma}(\check{\boldsymbol{T}})$ according to Equation (16) for the three designs are shown Figure 5 . The bound which was satisfied for both simultaneous and independent designs was not for sequential design and

Table 1. The parameters of PI controllers.

\begin{tabular}{ccccccc}
\hline Loop & \multicolumn{2}{c}{ Loop 1 } & \multicolumn{3}{c}{ Loop2 } \\
\hline PI parameters & $K_{p}$ & & $K_{i}$ & $K_{i}$ & $K_{p}$ & 0.692 \\
Sequential & 8.947 & 0.014 & 0.175 & 2.067 & 1.969 \\
Simultaneous & 3.0567 & 0.1000 & 5.566 & 0.726 \\
Independent & 8.947 & 0.014 & &
\end{tabular}

\begin{tabular}{|c|c|c|c|c|c|c|}
\hline \multirow{2}{*}{$\begin{array}{l}\text { Controlled variables } \\
\text { Performance metrics }\end{array}$} & \multicolumn{3}{|c|}{ Riser temperature (K) } & \multicolumn{3}{|c|}{ Regenerator cyclone temperature (K) } \\
\hline & $\begin{array}{l}\text { Rising time } \\
\text { (min) }\end{array}$ & $\begin{array}{l}\text { Settling time } \\
\text { (min) }\end{array}$ & $\begin{array}{c}\text { Overshoot } \\
\text { (\%) }\end{array}$ & $\begin{array}{l}\text { Rising time } \\
\text { (min) }\end{array}$ & $\begin{array}{l}\text { Settling time } \\
\text { (min) }\end{array}$ & $\begin{array}{c}\text { Overshoot } \\
\text { (\%) }\end{array}$ \\
\hline Sequential & 19.21 & 338.41 & 33.63 & 30.57 & 318.29 & 33.21 \\
\hline Simultaneous & 21.20 & 81.26 & 14.96 & 17.92 & 79.53 & 14.89 \\
\hline Independent & 20.34 & 98.79 & 19.56 & 16.50 & 99.54 & 15.87 \\
\hline
\end{tabular}

Table 2. Time domain performance metrics. 

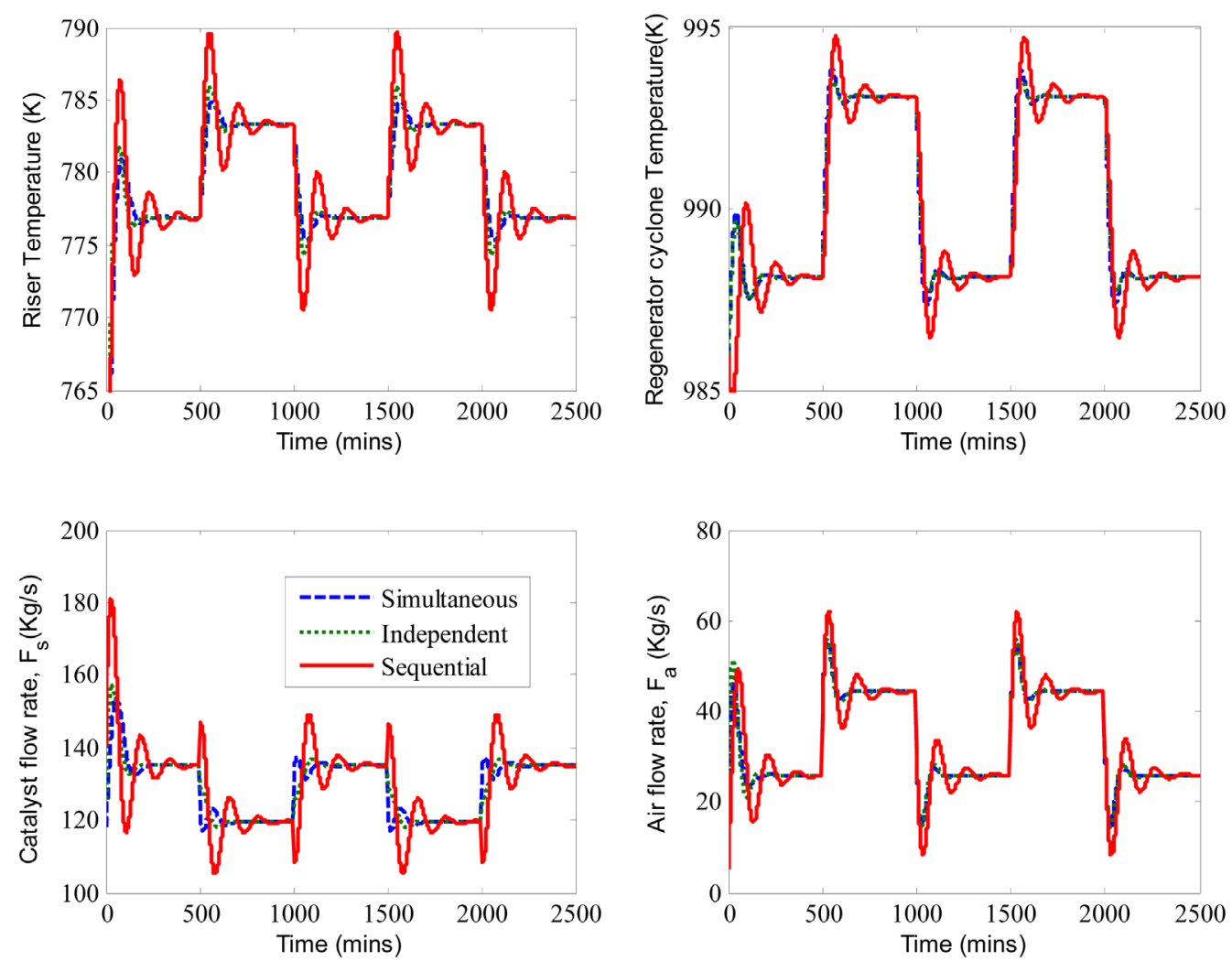

Figure 5. Closed-loop response of the FCCU sub-process for the three controller designs.

this implied that nominal stability (NS) was achieved for both simultaneous and independent designs and cannot be achieved for sequential design at certain range of frequencies.

However, independent design is more stable than simultaneous design as $\bar{\sigma}(\check{T})$ of independent design almost touches the bound at frequency around $0.1 \mathrm{rad} / \mathrm{min}$ which means its NS is marginal.

We examined the multivariable feedback loop consisting of the loop transfer matrix $\boldsymbol{L}=\boldsymbol{G K}$ for the three design. This was done to analyzed classical gain and phase margins corresponding to loop-at-a-time gain for each channel; and multiloop disk margin corresponding to simultaneous, independent, variations in the individual channels of loop transfer matrix in order to know the level of robust stability (RS).

Classical gain and phase margins give measure of the "safety margin" for closed-loop stability. Phase margin is a measure of how much phase variation is required at the gain crossover frequency to lose stability while gain margin is a measure of what relative gain variation is expected at the gain crossover frequency to lose stability. The larger the stability margins, the more stable the system is. The disk margin describes a circular region centered on the negative real axis at the average GainMargin (GM), e.g., $\left(\mathrm{GM}_{\text {low }}+\mathrm{GM}_{\text {high }}\right) / 2$ in a way that the loop transfer function $\mathrm{L}$ will not enter that region. The disk margin calculates the largest region for each channel such that for all gain and phase variations inside the region the nominal closed-loop system is stable. It suggests by how much transfer function of feedback channel under consideration can vary before this particular loop becomes unstable. In multiple margin analysis of the plant inputs, simultaneous, independent gain and phase margin variations are effected in each channel. Multiloop disk margin gives the largest region which keeps the closed-loop system stable for all gain and phase variations, occurring independently in each channel without getting outside the region. Both disk margins and multiloop disk margins bounds originate from the radius of the circle computed based on the balanced sensitivity function $S+T$. The bound is formulated as a $\mu$-analysis problem with each channel perturbed by an independent, complex perturbation. The peak $\mu(S+T)$ value ensures that any simultaneous, independent phase and gain variations carried out on each loop simultaneously will not make the system unstable if it lies inside the corresponding circle or disk of size $\mu(S+T)$.

The results are presented in Table 3 with the support of Robust toolbox in Matlab, all the designs gave infi- 
nite classical gain margin, and so no comparison is possible based on this information. In term of classical phase margin (PM), it appeared independent designs gave the best especially when looking at loop1 of the design which gave the largest PM of 82.90 degs at $0.56(\mathrm{rad} / \mathrm{min})$. Similar result was obtained for disk margin analysis, independent design gave largest variation as it indicates the closed-loop system will remain stable for simultaneous gain variations of [0.11 9.39] and phase margin variations of \pm 77.84 deg in the first input channel (loop1). The multivariable margin analysis led to a maximum allowable gain margin variation of [0.3469 2.8827] and phase margin variations of \pm 51.7374 deg indicating that the closed-loop system for independent design would remain stable for independent and simultaneous variations in gain and phase margins. The result obtained from classical PM and disk gain analysis for sequential design gave impression that it performed better than simultaneous design. However, when multiloop disk analysis was applied no value was obtained for gain margin and phase margin in the sequential design, indicating that stability could not be achieved for this design. The outcome of these analyses justified what was observed in Figure 6 in which independent design had best performance while the sequential design gave the worst performance in term of nominal stability.

We carried out robust stability tests by perturbing $20 \%$ uncertainty without making use of change in magnitude with frequency in our analyses. The worst gain margins analysis gives the largest disk margin such that the closed-loop system is stable for specified range of uncertainly set and all values of gain and phase variations that lie inside the disk. The worst-case gain and phase margin bounds which are computed based on the balanced sensitivity function indicate that the closed-loop system will be stable for a given uncertainty set and would remain stable for an additional gain and phase margin variation in the considered input/output channel. From Table 3, no margin is obtained for sequential design an indication of instability, however, independent design gives a maximum allowable gain margin variation and phase margin variations in both input channels in the presence of the uncertainties indicating its best performance in term of robust stability when compared to other two designs.

Finally we examined robust performance by looking at six perturbed plants randomly generated based on $20 \%$ uncertainty. The maximum singular values of the sensitivity functions for nominal and perturbed plants bounded by inverse of performance weight $\left(W_{P}\right)$ are shown in Figure 7. The sensitivity for the nominal plant $(S)$ is shown by the solid line, perturbed plant $\left(S_{p}\right)$ by dotted lines and inverse of performance weight $\left(W_{P}\right)$ by dashed line. It

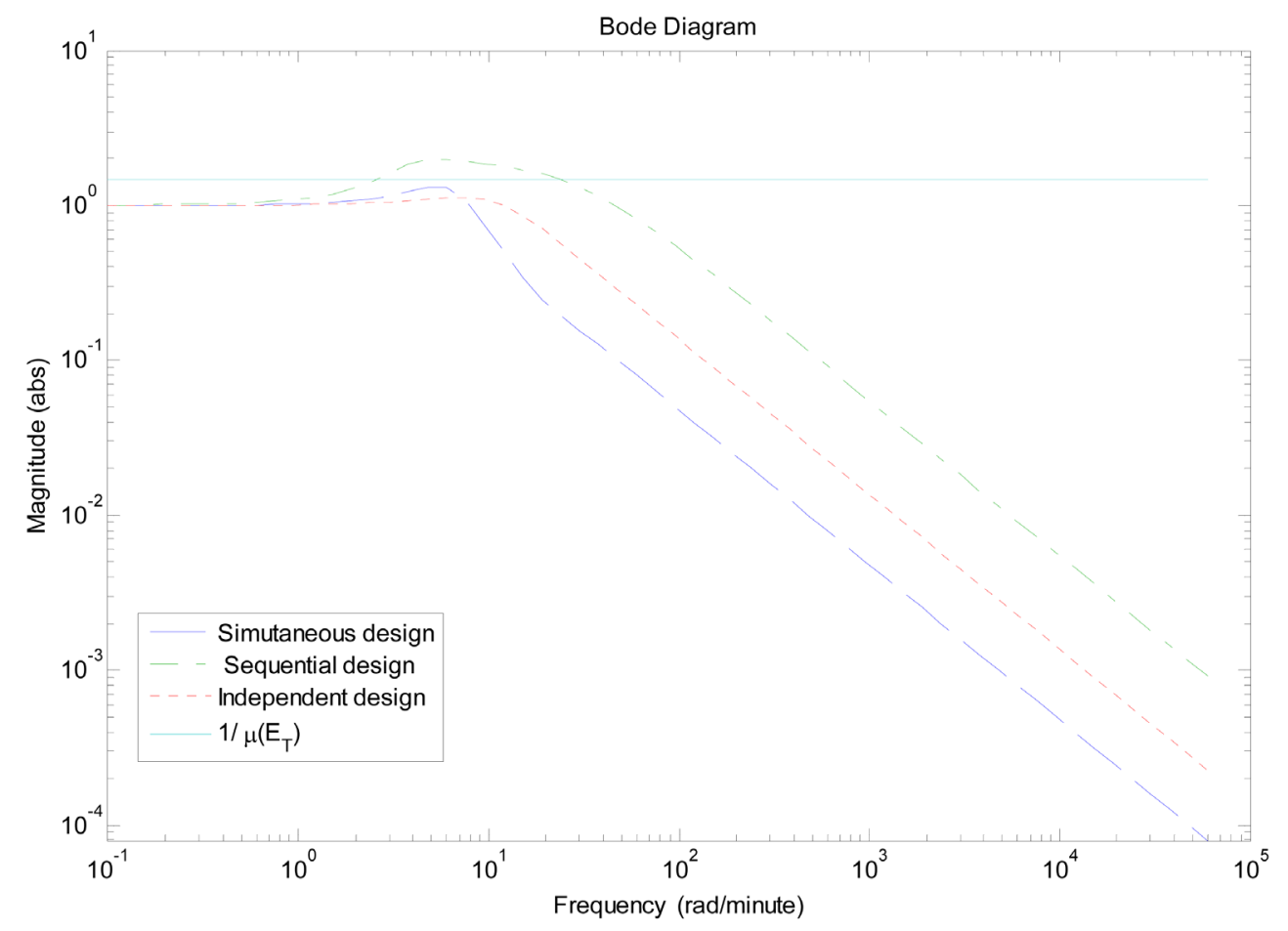

Figure 6. Bound on complementary sensitivity functions $\bar{\sigma}(\check{T})$. 

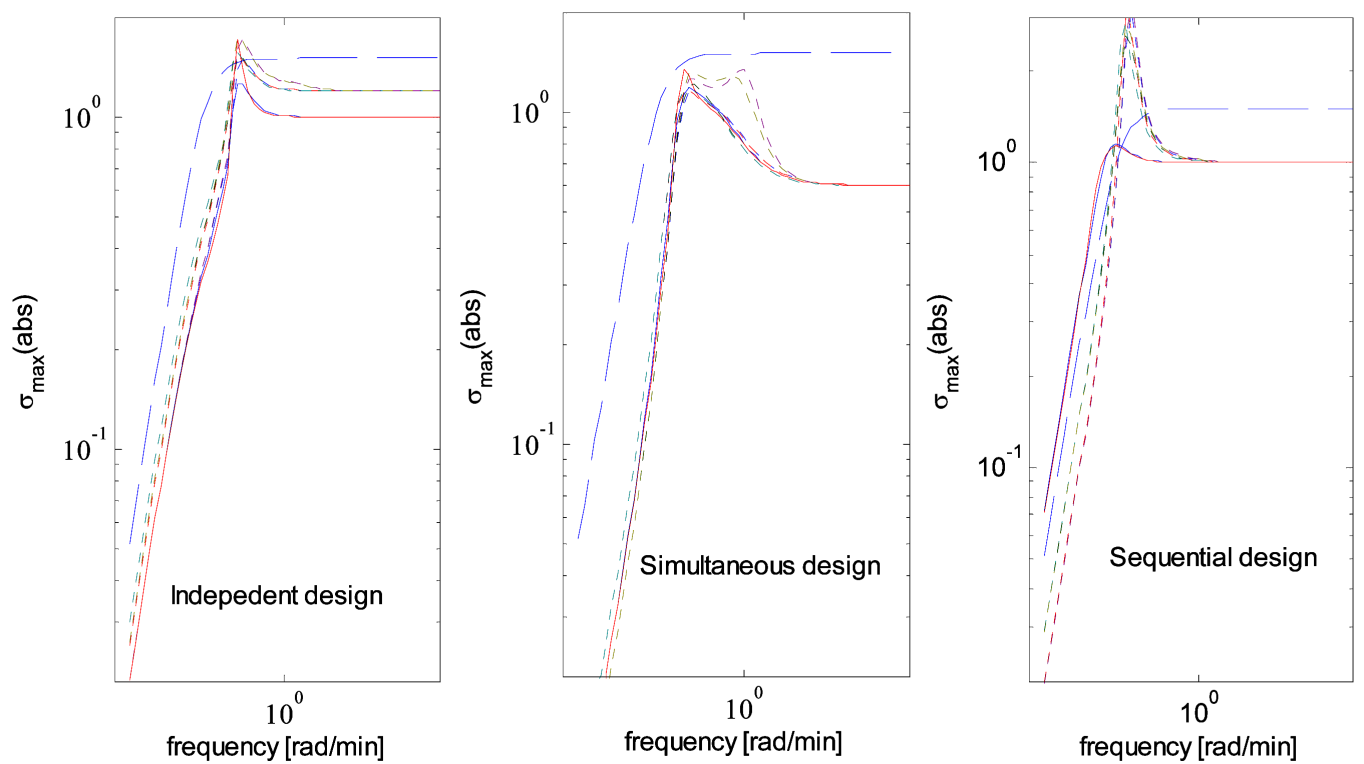

Figure 7. Maximum singular values (dashed line: inverse of performance weight $W_{p}$; solid line: nominal sensitivity function S; dotted lines: perturbed sensitivity functions $S_{P}$ ).

Table 3. Nominal and robust stability tests.

\begin{tabular}{|c|c|c|c|c|c|c|}
\hline & \multicolumn{2}{|c|}{ Sequential } & \multicolumn{2}{|c|}{ Independent } & \multicolumn{2}{|c|}{ Simultaneous } \\
\hline & Loop1 & Loop2 & Loop1 & Loop2 & Loop1 & Loop2 \\
\hline Classical GM & Inf, & Inf, & Inf, & Inf, & Inf, & Inf, \\
\hline GMf requency (rad/min) & Inf & Inf & Inf & Inf & Inf & $\operatorname{Inf}$ \\
\hline Classical PM (degs.) & 68.51 & 64.96 & 82.90 & 55.88 & 34.66 & 35.02 \\
\hline PMf requency (rad/min) & 0.11 & 0.11 & 0.56 & 0.07 & 0.04 & 0.05 \\
\hline Disc GM & {$\left[\begin{array}{ll}0.19 & 0.17\end{array}\right]$} & [0.23 4.47] & [0.12 9.39] & [0.31 3.27] & [0.54 1.87] & {$\left[\begin{array}{lll}0.53 & 0.90\end{array}\right]$} \\
\hline Disc PM and & \pm 68.21 & \pm 64.77 & \pm 77.84 & \pm 55.97 & \pm 33.70 & \pm 34.55 \\
\hline PMf requency (rad/min) & 0.11 & & 0.17 & 0.07 & 0.04 & 0.04 \\
\hline Multiple GM & Nil & Nil & \multicolumn{2}{|c|}{ [0.35 2.88] } & \multicolumn{2}{|c|}{$\left[\begin{array}{lll}0.70 & 1.43\end{array}\right]$} \\
\hline Multiple PM & Nil & Nil & \multirow{2}{*}{\multicolumn{2}{|c|}{$\begin{array}{l} \pm 51.74 \\
0.0854\end{array}$}} & \multirow{2}{*}{\multicolumn{2}{|c|}{$\begin{array}{l} \pm 20.05 \\
0.0431\end{array}$}} \\
\hline & & & & & & \\
\hline Worst case gain & Nil & Nil & [0.24 4.12] & [0.53 1.89] & [0.88 1.13] & {$\left[\begin{array}{lll}0.90 & 1.12\end{array}\right]$} \\
\hline Worst case phase & & & \pm 62.94 & \pm 34.14 & \pm 7.16 & \pm 6.69 \\
\hline
\end{tabular}

can be observed that the nominal and six perturbed plants are below the bound for simultaneous design implying that both NP and RP are satisfied for this design. In the case of independent design, NP and RP are almost satisfied because at certain frequency, both nominal plant and perturbed plants have peaks outside the bond. In the case of sequential design, there was poor performance because nominal plant and some perturbed plants were not bounded and the peaks were more pronounced. The sequential design was able to achieve a robust performance margin of 0.8143 implying $81.4 \%$ model uncertainty and a performance margin of 1.23 at $0.126 \mathrm{rad} / \mathrm{min}$. Simultaneous design gave a robust performance margin of 0.5896 to imply size of $59 \%$ for model uncertainty with 1.7 performance margin at $0.0794 \mathrm{rad} / \mathrm{min}$. In, Independent design a robust performance margin of 0.599 was achieved for the uncertainty system (59.9\% size) resulting to a performance margin of 1.67 at $0.01 \mathrm{rad} / \mathrm{min}$.

The "true" worst case weighted sensitivity, $\max S_{P}=\left\|\boldsymbol{W}_{P} \boldsymbol{S}_{p}\right\|_{\infty}$ were obtained as 1.04, 1.69 and 1.49 for simultaneous design, independent design and sequential design respectively. While the peak values of the sensitivity function for the corresponding worst case perturbed plant for simultaneous design, independent design and 
sequential design were respectively 1.33 at $0.02 \mathrm{rad} / \mathrm{min}, 1.13$ at $0.08 \mathrm{rad} / \mathrm{min}$ and 2.09 at $0.04 \mathrm{rad} / \mathrm{min}$.

\section{Conclusion}

In this paper, we compared three methods of design decentralized PI controllers for riser-regenerator subprocess of FCCU. We proposed an algorithm for constrained global optimization that combined the augmented Lagrangian technique for handling the equality and inequality constraints with a genetic algorithm as optimizer to tune controller parameters. This was achieved by minimizing infinity norm of weighted sensitivity function subject to constraint formulated by imposing $\mu$-interaction measure as bound on diagonal complementary sensitivity functions $\bar{\sigma}(\check{\boldsymbol{T}})$ to guarantee nominal stability. We observe that sequential design gives the worst behaviors' for both nominal and robust performances. We have found that decentralized control configurations with simple PI controllers can still give level of robustness in the presence of inaccurate knowledge of steady-state gains and time constants. An advantage of the proposed control design is that easy tuning procedures can be achieved for multivariable configuration. Furthermore, the compensator design procedure to avoid input saturation is relatively simple and can be implemented easily.

\section{References}

[1] Jose, A.R., Jesus, V. and Hector, P. (2004) Multivariable Control Configurations for Composition Regulation in a Fluid Catalytic Cracking Unit. Chemical Engineering Journal, 99, 187-120.

[2] Jose, R.H., Richart, V.R. and Daniel, S.S. (2006) Multiplicity of Steady States in FCC Units: Effect of Operating Conditions. Fuel, 85, 849-859.

[3] Pandimadevi, V., Indumathi, G. and Selvakumar, P. (2010) Design of Controllers for a Fluidized Catalytic Cracking Process. Chemical Engineering Research and Design, 8, 875-880.

[4] Iancu, M. and Agachi, P.S. (2010) Optimal Process Control and Operation of an Industrial Heat Integrated Fluid Catalytic Cracking Plant Using Model Predictive Control. In: Ferraris, S.P., Ed., 20th European Symposium on Computer Aided Process Engineering, Elsevier B.V. www.sciencedirect.com/science/article/pii/S1570794610280859

[5] Raji, O.Y., El-Nafaty, U.A., Jibril, M. and Danjuma, B.M. (2012) Modelling and Optimization of Fluid Catalytic Cracking Unit (FCCU) Using Hysys. International Journal of Emerging Trends in Engineering and Development, 3 , $1-9$.

[6] Ansari, R.M. and Tade, M. (2000) Constrained Nonlinear Multivariable Control of a Fluid Catalytic Cracking Process. Journal of Process Control, 10, 539-555.

[7] Raluca, R., Zoltán, K.N., Frank, A. and Serban, P.A. (2005) Dynamic Modeling and Nonlinear Model Predictive Control of a Fluid Catalytic Cracking Unit. In: Espuña, Ed., L.P., European Symposium on Computer-Aided Process Engineering-15, Elsevier Science B.V.

[8] Ahmed, D.F. (2011) Decoupling Control of Fluid Catalytic Cracking Unit. Journal of Chemistry and Chemical Engineering, 5, 12-19.

[9] Garcia, D., Karimi, A. and Longchamp, R. (2005) PID Controller Design for Multivariable Systems Using Gershgorin Bands. IFAC. www.nt.ntnu.no/users/skoge/prost/proceedings/ifac2005/.../02149.pd

[10] Rosinová, D., Thuan, N.Q. and Veselý, V.M. (2012) Robust Decentralized Controller Design: Subsystem Approach. Journal of Electrical Engineering, 63, 28-34.

[11] Skogested, S. and Postlethwaite, I. (2005) Multivariable Feedback Control Analysis and Design. John Wiley Sons, Chichester, New York, Brisbane, Toronto and Singapore.

[12] Campo, P.J. and Morari, M. (1994) Achievable Closed-Loop Properties of Systems under Decentralized Control: Conditions Involving the Steady-State Gain. IEEE Transactions on Automatic Control, 39, 932-943. http://dx.doi.org/10.1109/9.284869

[13] Skogestad, S. and Morari, M. (1989) Robust Performance of Decentralized Control Systems by Independent Designs. Automatica, 25, 119-125. http://dx.doi.org/10.1016/0005-1098(89)90127-1

[14] Hovd, M. and Skogestad, S. (1993) Procedure for Regulatory Control Structure Selection with Application to the FCC Process. AIChE Journal, 39, 1938-1953. http://onlinelibrary.wiley.com http://dx.doi.org/10.1002/aic.690391205

[15] Alsabei, R.M. (2011) Model Based Approach for the Plant-Wide Economic Control of Fluid Catalytic Cracking Unit. PhD Thesis, Loughborough University, Loughborough.

[16] McFarlane, R.C., Reineman, R.C., Bartee, J.F. and Georgakis, C. (1990) Dynamic Simulator for a Model IV Fluid Cat- 
alytic Cracking Unit. Proceedings of the American Institute of Chemical Engineers Annual Meeting, Chicago, 14-16 November 1990.

[17] Coleman, B. and Babu, J. (2002) Techniques of Model Based Control. Prentice Hall, Upper Saddle River.

[18] Zhou, K. (1999) Essentials of Robust Control. Pretence Hall, Upper Saddle River.

[19] Morari, M. (1983) Design of Resilient Processing Plants: A General Framework for the Assessment of Dynamic Resilience. Chemical Engineering Science, 38, 1881-1891. http://dx.doi.org/10.1016/0009-2509(83)85044-1

[20] Deb, K. and Srivastava, S. (2013) A Genetic Algorithm Based Augmented Lagrangian Method for Constrained Optimization. Computational Optimization and Applications, 53, 869-902.

[21] Lewis, R. and Torczon, V. (2002) A Globally Convergent Augmented Lagrangian Pattern Search Algorithm for Optimization with General Constraints and Simple Bounds. SIAM Journal on Optimization, 12, 1075-1089.

[22] Costa, L., Santo, A.E. and Fernandes, E.M. (2012) A Hybrid Genetic Pattern Search Augmented Lagrangian Method for Constrained Global Optimization. Applied Mathematics and Computation, 218, 9415-9426. http://dx.doi.org/10.1016/j.amc.2012.03.025

[23] Goldberg, D. (1989) Genetic Algorithms in Search, Optimization, and Machine Learning. Addison-Wesley, Reading, MA.

[24] Melanie, M. (1989) An Introduction to Genetic Algorithms. MIT Press, Cambridge. 